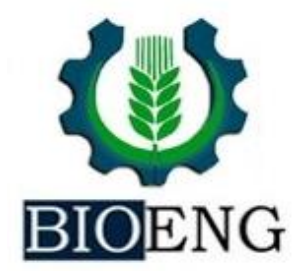

\title{
O IMPACTO DO DÉFICIT HÍDRICO E DO TRATAMENTO INDUSTRIAL DE SEMENTES NA GERMINAÇÃO, DESENVOLVIMENTO, VIGOR E PRODUÇÃO DE MATÉRIA SECA EM PLÂNTULAS DE SOJA
}

\author{
S. B. Pinheiro ${ }^{1 *}$, H. J. S. Costa ${ }^{1}$, J. M. Freitas ${ }^{1}$, P. R. Frade ${ }^{1,2}$, \\ A. A. Silva ${ }^{1}$, C. S. Pereira ${ }^{3}$ \\ ${ }^{1}$ UNIFOR - Centro Universitário de Formiga, Formiga, MG, Brasil \\ ${ }^{2}$ UFLA - Universidade Federal de Lavras, Lavras, MG, Brasil \\ ${ }^{3}$ UFMT - Universidade Federal de Mato Grosso, Sinop, MT, Brasil
}

Article history: Received 04 September 2019; Received in revised form 20 September 2019; Accepted 25 September 2019; Available online 30 September 2019.

\section{RESUMO}

O cultivo da soja exige um alto nível de qualidade em relação às sementes empregadas, além de ser muito dependente da condição hídrica. Dentro dessa sistemática, este estudo avaliou o impacto do déficit hídrico na germinação de sementes na cultura de soja. Foram avaliados 12 tratamentos, com 4 repetições em cada, totalizando 50 sementes de soja por repetição, em que se comparou o rendimento das sementes tratadas industrialmente com standak top $200 \mathrm{ml}$ a cada $100 \mathrm{~kg}$ de sementes (piraclostrobina $25 \mathrm{~g} / \mathrm{l}+$ tiofanato metílico 225g/l + fipronil 713g/l), com fungicidas em comparação com as sementes não tratadas. Os resultados obtidos evidenciaram que a ausência de água até 14 dias não impactou na produtividade e rendimento produtivo das plântulas. No entanto, após esse período, a ausência de umidade influenciou na qualidade fisiológica das sementes, comprometendo o stand final. As análises feitas acerca da influência da temperatura e umidade foram pouco significativas. Outra conclusão deste experimento foi que as sementes tratadas obtiveram um resultado aquém do obtido pelas sementes não tratadas. Pressupõe-se, a partir das análises feitas, que o tratamento empregado nas sementes tratadas industrialmente não tenha sido realizado de forma adequada, causando fitotoxidez na semente ou injúrias causadas no momento da inserção da mistura que comprometeram a qualidade fisiológica e química da semente tratada e, por essa razão, seu rendimento não sobrepujou o obtido pelas sementes não tratadas.

Palavras-chave: Pluviosidade; produtividade; sementes tratadas; sementes não tratadas.

\section{THE IMPACT OF WATER DEFICIT AND INDUSTRIAL TREATMENT OF SEEDS ON GERMINATION, DEVELOPMENT, VIGOR AND DRY MATTER PRODUCTION IN SOYBEAN SEEDLINGS}

\begin{abstract}
Soybean cultivation requires a high level of quality in relation to the seeds employed, besides being very dependent on the water condition. Within this systematic, this study evaluated the impact of water deficit on seed germination in soybean crop. Twelve treatments were evaluated, with 4 replications in each, totaling 50 soybean seeds per repetition, comparing the yield of the seeds treated industrially with standak top $200 \mathrm{ml}$ every $100 \mathrm{~kg}$ of seeds (Piraclostrobina 25g/L + thiofanate Methyl 225g/L + fipronil $713 \mathrm{~g} / \mathrm{L}$ ), with fungicides compared to untreated seeds. The results showed that the absence of water up to 14 days did
\end{abstract}

\footnotetext{
* saulobpengagro2015@gmail.com
} 
not impact on productivity and productive yield. However, after this period, the absence of moisture influenced the physiological quality of the seeds, compromising the final stand. The analyses made about the influence of temperature and humidity were not significants. Another conclusion of this experiment was that the treated seeds obtained a result below that obtained by untreated seeds. From the analyses performed, it is assumed that the treatment used in the industrially treated seeds has not been adequately performed, causing phytotoxicity in the seed or injuries caused at the time of the insertion of the mixture that compromised the Physiological and chemical quality of the treated seed and, for this reason, its yield did not overcome the obtained by untreated seeds.

Keywords: Rainfall; productivity; treated seeds; untreated seeds.

\section{INTRODUÇÃO}

A soja é considerada uma das principais oleaginosas produzida e consumida mundialmente. Essa representatividade se justifica por suas múltiplas aplicabilidades, tanto para o consumo animal, por meio do farelo de soja, quanto para o consumo humano, no formato de grão e óleo. No contexto brasileiro, a soja também possui grande relevância, em que se observa a expansão de seu cultivo para várias regiões do país, com destaque para o Cerrado (SILVA et al., 2011).

A mecanização e a criação de cultivares altamente produtivas adaptadas às diversas regiões; o desenvolvimento de pacotes tecnológicos relacionados ao manejo de solos; ao manejo de adubação balanceada com macro e micronutrientes; técnicas de correção de acidez; manejo integrado de pragas e doenças; processo de inoculação das sementes para fixação biológica do nitrogênio, além da identificação e solução para os principais aspectos responsáveis por perdas no processo de colheita, são fatores promotores desse avanço, permitindo que a cultura expresse sua potencialidade nas diversas condições edafoclimáticas do território brasileiro (FREITAS, 2011).

Graças à observância desses fatores, o Brasil se tornou o principal país exportador do produto.

Segundo a Embrapa Soja (2018), as perspectivas apontam para uma produção recorde no ano de 2019, com estimativas na casa de 121 milhões de toneladas, ultrapassando a marca histórica de 2018 em que o país produziu 118 milhões de toneladas e assumiu a primeira posição no ranking mundial de exportação do produto.

Para manter esse alto nível de produtividade, é preciso atentar para alguns aspectos intrínsecos ao respectivo cultivo.

O cultivo da soja exige um alto nível de qualidade em relação às sementes empregadas. Observa-se que os quatro componentes básicos que determinam a qualidade das sementes, fisiológicos, genéticos, sanitários e físicos, apresentam importância análoga. Porém, vale salientar a influência exercida pelo potencial fisiológico que contribui para a obtenção de alta produtividade e por oportunizar que o produtor avalie in loco o desempenho das sementes adquiridas (MARCOS FILHO, 2013).

Dentre os aspectos destacados por Marcos Filho (2013), a emergência rápida e uniforme das plântulas colabora de maneira incisiva para que se obtenha a população desejada de plantas por área.

Verifica-se também que diversos fatores abióticos interferem no desenvolvimento da soja, como climáticos e edáficos. A ação de muitos desses fatores é responsável por restringir ou inibir o desenvolvimento da cultura da soja. Por essa razão, é preciso avaliar a temperatura, o fotoperíodo, a pluviosidade e o tipo de solo (MILANESI, 2015).

Dentre esses fatores que influenciam na produtividade, Machado et al. (2016) enfatizam $o$ papel exercido pelas adversidades climáticas, uma vez que elas 
interferem de forma sistemática na produtividade do cultivo da soja, especialmente, durante a fase de estabelecimento do stand inicial dessa cultura. Dentre os estresses que limitam sua produtividade, destaca-se o déficit hídrico, de maneira mais contundente, durante a fase de germinação. Por essa razão, é imprescindível que sejam assegurados os índices hídricos necessários para cada fase do respectivo cultivo.

Essa dependência hídrica é justificada uma vez que a soja "possui $90 \%$

\section{MATERIAIS E MÉTODOS}

O trabalho foi conduzido e realizado em uma casa de vegetação, localizada na Fazenda Laboratório do Centro Universitário de Formiga (UNIFOR-MG) latitude $20^{\circ} 27^{\prime \prime} 58.9$ " S" e longitude de $45^{\circ} 28^{\prime \prime} 0.7$ "w" e 944 metros de altitude.

Foram avaliados os impactos do déficit hídrico em 12 tratamentos, com 4 repetições em cada, totalizando 50 sementes de soja por repetição. Dos 12 tratamentos realizados, 6 foram efetuados com o tratamento industrial de sementes e 6 tratamentos sem o tratamento industrial. O plantio foi realizado em bandejas com 50 células com medidas externas de 545 $\mathrm{mm}$ x $280 \mathrm{~mm}$, profundidade de $100 \mathrm{~mm}$ e volume total de $90 \mathrm{~cm}^{3}$ por célula.

As sementes utilizadas foram do tipo cultivar Monsoy 6410 I PRO intacta safra 2017/2018, categoria S2, pureza $100 \%$, germinação $96 \%$, com tratamento de seu peso formado de água, cuja importância é fundamental para processos fisiológicos e bioquímicos, sendo indispensável para o pleno desenvolvimento da cultura" (MILANESI, 2015, p. 19).

Dentro do contexto apresentado, o presente trabalho tem como objetivo avaliar o impacto do déficit hídrico na germinação de sementes na cultura de soja, bem como, os malefícios ou benefícios do tratamento industrial de sementes.

industrial de sementes standak top $200 \mathrm{ml}$ a cada $100 \mathrm{~kg}$ de sementes (piraclostrobina $25 \mathrm{~g} / 1$ + tiofanato metílico $225 \mathrm{~g} / 1+$ fipronil $713 \mathrm{~g} / \mathrm{l})$, com teste de germinação em caixa de areia apontando 94\% de germinação. As sementes sem o tratamento industrial foram cultivar Monsoy 6410 I PRO com germinação em torno de $98 \%$.

O solo utilizado no experimento foi um latossolo vermelho eutrófico, coletado em uma área com histórico de uso de 7 anos com plantio da cultura da soja e sobrotação com outras culturas.

O solo passou por análise de fertilidade, apresentando teor inicial de água presente no mesmo 3,3\% de $\mathrm{U} \%$, demonstrada por meio da Tabela 1. Foram adicionados $20 \%$ de substrato Mococa inerte a base de turfa, casca de pinus e carvão vegetal para melhor condicionamento e estruturação do solo do experimento.

Tabela 1 - Laudo de análise do solo

\begin{tabular}{|c|c|c|}
\hline ELEMENTO QUÍMICO & UNIDADE DE MEDIDA & RESULTADO \\
\hline $\mathrm{pH} \mathrm{CaCl}{ }_{2}$ & $1: 2,5$ & 6,5 \\
\hline $\mathrm{P} \mathrm{Meh}^{-1}$ & $\mathrm{mg} \mathrm{dm}^{-3}$ & 22,8 \\
\hline P rem. & $\mathrm{mg} \mathrm{dm} \mathrm{dm}^{-3}$ & 19,4 \\
\hline K Mehlich & $\mathrm{mg} \mathrm{dm}{ }^{-3}$ & 188,82 \\
\hline $\mathrm{S}-\mathrm{SO}_{4}^{-2}$ & $\mathrm{mg} \mathrm{dm}{ }^{-3}$ & 12 \\
\hline $\mathrm{Ca}^{2+}$ & mmolc $\mathrm{dm}^{-3}$ & 60,0 \\
\hline $\mathrm{Mg}^{2+}$ & mmolc $\mathrm{dm}^{-3}$ & 15,0 \\
\hline $\mathrm{Al}^{3+}$ & mmolc $\mathrm{dm}^{-3}$ & 0,00 \\
\hline $\mathrm{H}^{+} \mathrm{Al}$ & mmolc $\mathrm{dm}^{-3}$ & 11,00 \\
\hline MO & $\mathrm{g} / \mathrm{kg}$ & 26,9 \\
\hline $\mathrm{CO}$ & $\mathrm{g} / \mathrm{kg}$ & 15,6 \\
\hline
\end{tabular}




\begin{tabular}{ccc}
\hline SB & mmolc dm & \\
t & mmolc dm & 79,80 \\
CTC & mmolc dm & 79,80 \\
V & $\%$ & 90,80 \\
m & $\%$ & 88 \\
\hline
\end{tabular}

Fonte: Dados da Pesquisa, 2019.

Nota: Dados obtidos por meio da Agrolab Análises Agrícolas, 2019.

Os tratamentos foram semeados em 31 de outubro de 2018, com profundidade de $3 \mathrm{~cm}$. O uso de um paquímetro digital foi empregado para minimizar a variância da profundidade de semeadura.

Os 12 tratamentos foram divididos em 6 parcelas em que a testemunha recebeu a irrigação logo após o término do plantio. Os demais tratamentos recebendo irrigação sequencialmente de acordo com a com a respectiva divisão:

Tratamento 1 - (T1) com irrigação 7 dias após a semeadura;

Tratamento 2 - (T2) com irrigação 14 dias após o plantio;

Tratamento 3 - (T3) com irrigação 21 dias após o plantio;

Tratamento 4 - (T4) com irrigação 28 dias após o plantio; e

Tratamento 5 - (T5) com irrigação 35 dias após o plantio.

A irrigação foi realizada com pulverizador de alta pressão com capacidade para 5 litros marca Guarani. Após a primeira irrigação, foi mantida a umidade na capacidade de campo de $60 \%$, cerca de 2,7 litros de água por repetição do experimento de acordo com o volume de solo e sua capacidade de retenção e armazenamento de água.

Os tratamentos foram avaliados por 14 dias subsequentes a data da primeira irrigação em que foram observados parâmetros como: índice de velocidade de emergência das sementes, comprimento da parte aérea, comprimento da raiz, matéria verde e matéria seca.

\section{RESULTADOS E DISCUSSÃO}

A Tabela 2 traduz o resumo da análise de variância referente ao experimento sobre o impacto do déficit hídrico e o tratamento industrial de
Para o cálculo do índice de velocidade de emergência foi realizada a contagem das plantas emergentes diariamente até a sua estabilização. As medições do comprimento da parte aérea e raiz foram realizadas com o auxílio de um paquímetro digital. Já, para a obtenção das matérias verde e seca, utilizou-se uma balança de precisão Shimadzu e uma estufa Odontobras na temperatura de $60^{\circ} \mathrm{C}$ durante 3 (três) dias para a secagem das plântulas.

Os dados climatológicos monitorados ao longo do experimento, como temperatura e umidade relativa foram obtidos pelo termômetro de casinha em dois intervalos de tempo específicos: manhã e tarde.

Os dados obtidos foram submetidos às análises estatísticas descritivas (média, erro padrão da média, desvio padrão, primeiro quartil, medianas e terceiro quartil).

A análise de variância Anova, a qual Minitab testa a hipótese das médias de duas ou mais populações serem iguais, testando a importância de um ou mais fatores comparando as médias das variáveis de resposta em diferentes níveis dos fatores, em que a hipótese nula afirma que pelo menos uma é diferente. Foi adotado nível de significância de $5 \%$.

O software utilizado para o desenvolvimento das respectivas análises estatísticas foi o SISVAR 5.0. Foi feito o teste Scott-Knott (1974).

sementes sobre a Emergência, índice de velocidade de emergência (IVE), comprimento de parte aérea (PA), comprimento de raiz (RAIZ), peso de 
matéria verde (MV) e peso de matéria seca (MS).

Os respectivos dados foram obtidos no ensaio semeadura a seco $\mathrm{x}$ tratamento industrial de sementes, ano agrícola 2018/ 2019, Centro Universitário de Formiga MG, UNIFOR-MG.

Tabela 2 - Resumo da análise de variância

\begin{tabular}{l|c|c|c|c|c|c|c}
\hline \multirow{2}{*}{ FV } & \multirow{2}{*}{ GL } & \multicolumn{7}{c}{ QUADRADOS MÉDIOS } \\
\cline { 3 - 8 } & & Emergência & IVE & PA & RAIZ & MV & MS \\
\hline Tratamento (A) & 1 & $1026,75^{*}$ & $556,10^{*}$ & $2,91^{*}$ & $8,84^{*}$ & $8,25^{*}$ & $0,17^{*}$ \\
Dias (B) & 5 & $979,88^{*}$ & $1295,90^{*}$ & $22,28^{*}$ & $9,66^{*}$ & $1312,85^{*}$ & $55,13^{*}$ \\
A x B & 5 & $176,95^{*}$ & $54,84^{*}$ & $0,80^{*}$ & $0,97^{*}$ & $233,62^{*}$ & $10,72^{*}$ \\
Resíduo & 36 & 30,36 & 5,58 & 0,57 & 0,28 & 11,10 & 0,40 \\
CV (\%) & & 6,49 & 7,36 & 7,49 & 5,15 & 10,60 & 9,96 \\
\hline
\end{tabular}

Fonte: Dados da Pesquisa, 2019.

Nota: *Significativo a 5\% Scott-Knott pelo teste F.

Neste experimento, foi analisada a influência da temperatura sobre o respectivo cultivo. Por meio das análises realizadas, observa-se que a temperatura teve pouca influência em função das médias terem tido pouca variação.
Em relação a umidade, constatou-se o mesmo resultado, ou seja, pouca variação.

Por meio da Tabela 3 é possível observar os resultados pertinentes à análise das variáveis temperatura e umidade.

Tabela 3 - Análise das variáveis: temperatura e umidade

\begin{tabular}{c|c|c|c}
\hline TRATAMENTOS & DIAS & TC $^{\mathbf{O}}$ MÉDIA & U\% RELATIVA \\
\hline T0 & 0 & 25,35 & 78,05 \\
T1 & 7 & 25,00 & 81,20 \\
T2 & 14 & 25,15 & 77,85 \\
T3 & 21 & 25,80 & 75,05 \\
T4 & 28 & 26,40 & 72,30 \\
T5 & 35 & 27,80 & 78,10 \\
\hline
\end{tabular}

Fonte: Dados da Pesquisa, 2019.

A seguir foram apresentados os resultados referentes às variáveis em estudo: Emergência, IVE, PA, RAIZ, MV e MS. Foram demonstrados os seis tratamentos, em que para cada um deles se analisou o resultado da semente tratada industrialmente, da semente não tratada e a média obtida em ambos os casos.
A título comparativo estabeleceu-se a média final, procurando denotar o tipo de semente que obteve melhor rendimento de acordo com as variáveis empregadas no experimento.

A primeira variável analisada foi a Emergência, sendo os respectivos resultados ilustrados por meio da Tabela 4 a seguir. 
Tabela 4 - Análise da Emergência: tratamento industrial e déficit hídrico

\begin{tabular}{|c|c|c|c|c|c|c|c|c|}
\hline \multirow[t]{2}{*}{ FV } & \multirow[t]{2}{*}{ Tratamento } & \multicolumn{7}{|c|}{ DIAS } \\
\hline & & 0 & 7 & 14 & 21 & 28 & 35 & MÉDIA \\
\hline \multirow{3}{*}{ Emergência } & Tratada & $\begin{array}{c}89,50 \\
\mathrm{aA}\end{array}$ & $\begin{array}{c}91,50 \\
\mathrm{aA}\end{array}$ & $\begin{array}{c}92,00 \mathrm{a} \\
\text { A }\end{array}$ & $\begin{array}{c}83,00 \mathrm{~b} \\
\mathrm{~B}\end{array}$ & $\begin{array}{c}52,50 \mathrm{~d} \\
\text { B }\end{array}$ & $\begin{array}{c}73,50 \mathrm{C} \\
B\end{array}$ & $80,33 \mathrm{~B}$ \\
\hline & Não tratada & $\begin{array}{c}93,00 \mathrm{a} \\
\mathrm{A}\end{array}$ & $\begin{array}{c}94,50 \\
\mathrm{aA}\end{array}$ & $\begin{array}{c}90,00 \\
\mathrm{aA}\end{array}$ & $\begin{array}{c}96,50 \\
\mathrm{aA}\end{array}$ & $\begin{array}{c}76,00 \\
\text { bA }\end{array}$ & $\begin{array}{c}87,50 \\
\mathrm{aA}\end{array}$ & $89,58 \mathrm{~A}$ \\
\hline & Média & $91,25 \mathrm{a}$ & 93,00 & $91,00 \mathrm{a}$ & $89,75 \mathrm{a}$ & $64,25 \mathrm{c}$ & $80,50 \mathrm{~b}$ & 84,96 \\
\hline
\end{tabular}

Fonte: Dados da Pesquisa, 2019.

Nota: Médias seguidas por letras minúsculas nas linhas e maiúsculas nas colunas não diferem entre si pelo teste de Scott-Knott de probabilidade.

Campos (2000) enfatiza a importância de se avaliar o número médio de plântulas, após a Emergência, para se obter dados mensuráveis que sejam capazes de auxiliar na tomada de decisão de se tratar ou não os lotes de sementes.

Nesse sentido, ao analisar a primeira variável em estudo, ou seja, as médias referentes à Emergência observa-se que em condições normais de campo, após a semeadura, as sementes absorvem água e começam a crescer. A radícula é a primeira a se alongar, seguida pelo coleóptilo, com plúmula incluída. Esse estádio, conhecido como VE ou emergência é atingido pela rápida elongação do mesocótilo, o qual empurra o coleóptilo em crescimento para a superfície do solo.

No presente trabalho, a interação entre semeadura de sementes tratadas e intervalo de tempo sem presença de água para início de processos metabólicos de germinação mostraram que os tratamentos: 0,7 e 14 dias não se diferiram estatisticamente para Emergência e apresentaram os melhores resultados comparados com os demais tratamentos. $\mathrm{O}$ tratamento 28 dias apresentou o pior resultado comparado com os demais. Em relação às sementes que não foram tratadas industrialmente, os melhores resultados foram constatados nos tratamentos $0,7,14$ e 21 dias, em que não se constatou grandes diferenças entre eles. $\mathrm{O}$ tratamento 28 dias também apresentou o pior resultado comparado com os demais.

Em síntese, ao comparar a evolução das sementes tratadas e não tratadas industrialmente, observa-se que a média final entre elas demonstra que as sementes não tratadas obtiveram um melhor desempenho em relação as que foram submetidas ao tratamento industrial.

Ao comparar esses resultados com outro experimento análogo, observa-se que os resultados foram um pouco diferentes, uma vez que a Emergência das sementes tratadas industrialmente apresentaram padrões semelhantes aos encontrados pela testemunha sem o tratamento (DAN et al., 2011).

Em outro estudo, observou-se que o tratamento industrial com fungicida permitiu uma melhor Emergência, confirmando que o tratamento permite uma maior proteção às sementes. No entanto, esse resultado é alterado quando as sementes permanecem tratadas por um período maior que quatro meses, pois o desempenho, nesse caso, obteve um resultado abaixo dos demais resultados evidenciados pelo experimento (KROHN; MALAVASI, 2004).

A partir da reflexão dos autores supracitados, é possível intuir que as sementes abordadas neste experimento ou foram tratadas de forma inadequada ou permaneceram em armazenamento num período superior ao período considerado ideal.

A segunda variável abordada neste experimento foi o índice de velocidade de emergência (IVE), obtido por meio da fórmula de Maguire que é a soma da germinação diária média.

Os respectivos resultados sobre o IVE foram sintetizados por meio da Tabela 5. 
Tabela 5 - Análise do IVE: tratamento industrial e déficit hídrico

\begin{tabular}{c|c|ccc|c|c|c|c|c}
\hline \multirow{2}{*}{ FV } & Tratamento & \multicolumn{8}{c}{ DIAS } \\
\cline { 3 - 9 } & & 0 & 7 & 14 & 21 & 28 & 35 & MÉDIA \\
\hline \multirow{4}{*}{ IVE } & Tratada & $39,15 \mathrm{bB}$ & 45,02 & 30,17 & 32,10 & 14,66 & 9,27 & $28,68 \mathrm{~B}$ \\
& & & $\mathrm{aB}$ & $\mathrm{cA}$ & $\mathrm{cB}$ & $\mathrm{dB}$ & $\mathrm{eB}$ & \\
& & Não tratada & 45,47 & 49,50 & 31,89 & 41,39 & 28,49 & 17,92 & $35,49 \mathrm{~A}$ \\
& & $\mathrm{bA}$ & $\mathrm{aA}$ & $\mathrm{dA}$ & $\mathrm{cA}$ & $\mathrm{dA}$ & $\mathrm{eA}$ & \\
& & Média & $42,31 \mathrm{~b}$ & $47,26 \mathrm{a}$ & $31,03 \mathrm{~d}$ & $36,75 \mathrm{c}$ & $21,58 \mathrm{e}$ & $13,60 \mathrm{f}$ & 32,09 \\
\hline
\end{tabular}

Fonte: Dados da Pesquisa, 2019.

Nota: Médias seguidas por letras minúsculas nas linhas e maiúsculas nas colunas não diferem entre si pelo teste de Scott-Knott de probabilidade.

Vale destacar que a qualidade fisiológica das sementes é considerada um fator básico de grande influência para o êxito da cultura da soja (Glycine max (L.) Merrill) (DIAS \& MARCOS FILHO, 1996). Em função disso, o IVE obtido a partir do teste de germinação é um parâmetro relevante para fins de produção de sementes de qualidade.

Por meio da comparação dessa variável, a partir dos resultados advindos das sementes tratadas industrialmente e não tratadas, observa-se que o tratamento 14 dias foi o único que não apresentou variação estatística significativa entre elas. Porém, nos demais tratamentos, a média final obtida evidencia que as sementes não tratadas obtiveram melhores resultados do que as que receberam o tratamento industrial.

Esse resultado foi obtido também por Dan et al. (2010), em que os autores observaram que a IVE obteve um resultado inferior nas sementes tratadas industrialmente pelos inseticidas carbofuran, [imidacloprid + thiodicarb] e acefato.

Posteriormente, foi desenvolvido um novo experimento, no qual os resultados não apresentaram diferença significativa entre o IVE das sementes tratadas industrialmente e não tratadas (DAN et al., 2011).

A terceira variável analisada pode ser verificada por meio da Tabela 6 , em que foi analisada a parte aérea (PA) da cultura da soja. Vale lembrar que ela é um importante parâmetro que serve para avaliar o desenvolvimento da cultura frente ao modelo de produção adotado juntamente com as características da área de produção, como fertilidade do solo, precipitação e clima da região produtora.

Tabela 6 - Análise da PA: tratamento industrial e déficit hídrico

\begin{tabular}{|c|c|c|c|c|c|c|c|c|}
\hline \multirow[t]{2}{*}{ FV } & \multirow[t]{2}{*}{ Tratamento } & \multicolumn{7}{|c|}{ DIAS } \\
\hline & & 0 & 7 & 14 & 21 & 28 & 35 & MÉDIA \\
\hline \multirow{5}{*}{ PA } & Tratada & 9,32 & 10,77 & 12,29 & 10,85 & 8,56 & 7,09 & $9,88 \mathrm{~B}$ \\
\hline & & $\mathrm{cA}$ & bA & $\mathrm{aA}$ & bA & $\mathrm{cA}$ & $\mathrm{dB}$ & \\
\hline & Não tratada & 9,64 & 11,12 & 12,68 & 11,08 & 9,64 & 8,46 & $10,37 \mathrm{~A}$ \\
\hline & & $\mathrm{cA}$ & $\mathrm{bA}$ & $\mathrm{aA}$ & $\mathrm{bA}$ & $\mathrm{cA}$ & $\mathrm{dA}$ & \\
\hline & Média & $9,48 \mathrm{~d}$ & $10,95 \mathrm{c}$ & $12,48 \mathrm{a}$ & $10,96 \mathrm{~b}$ & $9,10 \mathrm{e}$ & $7,78 \mathrm{f}$ & 10,12 \\
\hline
\end{tabular}

Fonte: Dados da Pesquisa, 2019.

Nota: Médias seguidas por letras minúsculas nas linhas e maiúsculas nas colunas não diferem entre si pelo teste de Scott-Knott de probabilidade.

Os resultados apresentados por meio da Tabela 6 demonstraram na comparação final entre os tipos de sementes analisadas, que as que receberam tratamento industrial obtiveram pior média em comparação com as sementes não tratadas.

Nesse comparativo, o tratamento 35 dias foi o único que se diferiu dos demais estatisticamente apresentando os piores 
resultados para sementes tratadas. Os demais tratamentos não apresentaram discrepâncias significativas, porém na conclusão final, foi evidenciado que as sementes que não receberam tratamento industrial apresentaram melhores resultados.

Conforme Vieira et al. (2013), os cultivares de soja, submetidos à déficit hídrico, apresentaram redução significativa do comprimento da PA. Dessa forma, observa-se a relação entre o potencial osmótico e o comprimento da PA, ou seja, quanto mais negativo o potencial osmótico, menores serão os valores de comprimento de PA.

No experimento desenvolvido por Dan et al. (2011), o tratamento industrial causou redução em relação ao acúmulo de MS da PA.

Em ambos os trabalhos descritos anteriormente apresentaram resultados similares ao presente estudo, ratificando os achados deste experimento em relação ao parâmetro analisado: PA.

A quarta variável em estudo foi a RAIZ, descrita na próxima tabela. Assim, por meio da Tabela 7, constata-se que os tratamentos 0,28 e 35 dias foram os únicos que apresentaram divergências significativas. Os piores resultados identificados ratificaram que as sementes tratadas não obtiveram o mesmo patamar de eficiência das sementes não tratadas.

Tabela 7 - Análise da RAIZ: tratamento industrial e déficit hídrico

\begin{tabular}{|c|c|c|c|c|c|c|c|c|}
\hline \multirow{2}{*}{ FV } & \multirow{2}{*}{ Tratamento } & \multicolumn{7}{|c|}{ DIAS } \\
\hline & & 0 & 7 & 14 & 21 & 28 & 35 & MÉDIA \\
\hline \multirow{5}{*}{ RAIZ } & Tratada & 9,49 & 10,94 & 11,44 & 10,79 & 8,61 & 8,07 & $9,89 \mathrm{~B}$ \\
\hline & & $\mathrm{bB}$ & $\mathrm{aA}$ & $\mathrm{aA}$ & $\mathrm{aA}$ & $\mathrm{cB}$ & $\mathrm{cB}$ & \\
\hline & Não tratada & 11,23 & 11,06 & 11,65 & 11,18 & 10,06 & 9,30 & $10,75 \mathrm{~A}$ \\
\hline & & $\mathrm{aA}$ & $\mathrm{aA}$ & $\mathrm{aA}$ & $\mathrm{aA}$ & $\mathrm{bA}$ & $\mathrm{bA}$ & \\
\hline & Média & $10,36 \mathrm{c}$ & $11,00 \mathrm{~b}$ & $11,54 \mathrm{a}$ & $10,99 \mathrm{~b}$ & $9,33 \mathrm{~d}$ & $8,69 \mathrm{e}$ & 10,32 \\
\hline
\end{tabular}

Fonte: Dados da Pesquisa, 2019.

Nota: Médias seguidas por letras minúsculas nas linhas e maiúsculas nas colunas não diferem entre si pelo teste de Scott-Knott de probabilidade.

Confirmando os achados dessa pesquisa, foram constatados também efeitos negativos provenientes do tratamento das sementes industrialmente, em que o comprimento de plântulas e RAIZ obteve um resultado aquém quando comparado com os resultados obtidos pelas sementes sem tratamento (DAN et al., 2010).

Em outro experimento, apurou-se o mesmo resultado, em que o tratamento industrial com inseticidas influenciou negativamente o crescimento da RAIZ das plântulas de soja (DAN et al., 2011).
Porém, o tratamento industrial, aplicado às sementes no experimento realizado por Cunha et al. (2015), não interferiu no comprimento da RAIZ das plântulas em estudo. Esses resultados são percebidos também por Guedes et al. (2009), reforçando ainda mais a constatação de que o tratamento industrial não interfere no comprimento da RAIZ.

A quinta variável analisada é a matéria verde (MV), descrita por meio da Tabela 8. 
Tabela 8 - Análise da MV: tratamento industrial e déficit hídrico

\begin{tabular}{|c|c|c|c|c|c|c|c|c|}
\hline \multirow{2}{*}{ FV } & \multirow{2}{*}{ Tratamento } & \multicolumn{7}{|c|}{ DIAS } \\
\hline & & 0 & 7 & 14 & 21 & 28 & 35 & MÉDIA \\
\hline \multirow{5}{*}{ MV } & Tratada & 41,32 & 34,65 & 32,56 & 27,45 & 11,30 & 17,98 & 31,02 \\
\hline & & $\mathrm{aB}$ & $\mathrm{bA}$ & $\mathrm{bA}$ & $\mathrm{cB}$ & $\mathrm{eB}$ & $\mathrm{dB}$ & $\mathrm{aA}$ \\
\hline & Não tratada & 62,19 & 37,66 & 35,41 & 34,13 & 19,05 & 23,52 & 31,85 \\
\hline & & $\mathrm{aA}$ & bA & bA & bA & $\mathrm{cA}$ & $\mathrm{cA}$ & $\mathrm{aA}$ \\
\hline & Média & $51,75 \mathrm{a}$ & $36,15 \mathrm{~b}$ & $33,98 \mathrm{~b}$ & $30,79 \mathrm{c}$ & $15,17 \mathrm{e}$ & $20,75 \mathrm{~d}$ & 31,43 \\
\hline
\end{tabular}

Fonte: Dados da Pesquisa, 2019.

Nota: Médias seguidas por letras minúsculas nas linhas e maiúsculas nas colunas não diferem entre si pelo teste de Scott-Knott de probabilidade.

Por meio da análise dos respectivos resultados, evidenciados por meio da Tabela 8, observa-se que o melhor resultado obtido foi o tratamento 0 tanto para as sementes tratadas quanto não tratadas. Já os piores resultados, foi o tratamento 28 dias para as sementes tratadas e os tratamentos 28 e 35 dias para as sementes não tratadas. Ainda segundo a variável MV em relação à comparação entre sementes tratadas e não tratadas industrialmente, a média final não apresentou distorções significativas entre elas.

A última variável em análise foi a matéria seca (MS), apresentada na tabela subsequente.

Tabela 9 - Análise da MS: tratamento industrial e déficit hídrico

\begin{tabular}{|c|c|c|c|c|c|c|c|c|}
\hline \multirow{2}{*}{ FV } & \multirow{2}{*}{ Tratamento } & \multicolumn{7}{|c|}{ DIAS } \\
\hline & & 0 & 7 & 14 & 21 & 28 & 35 & MÉDIA \\
\hline \multirow{4}{*}{ MS } & Tratada & 12,81 & 7,27 & 6,52 & 5,50 & 2,26 & 3,60 & $6,32 \mathrm{~A}$ \\
\hline & Não tratada & $\begin{array}{c}\mathrm{aA} \\
830\end{array}$ & $\begin{array}{l}\mathrm{bA} \\
753\end{array}$ & $\begin{array}{l}\mathrm{bA} \\
726\end{array}$ & $\begin{array}{c}\mathrm{cB} \\
683\end{array}$ & $\begin{array}{c}\mathrm{eB} \\
381\end{array}$ & $\begin{array}{c}\mathrm{dB} \\
493\end{array}$ & $644 \mathrm{~A}$ \\
\hline & & $\mathrm{aB}$ & $\mathrm{bA}$ & $\mathrm{bA}$ & $\mathrm{bA}$ & $\mathrm{dA}$ & $\mathrm{cA}$ & \\
\hline & Média & $10,56 \mathrm{a}$ & $7,40 \mathrm{~b}$ & $6,89 \mathrm{~b}$ & $6,16 \mathrm{c}$ & $3,03 \mathrm{e}$ & $4,26 \mathrm{~d}$ & 6,38 \\
\hline
\end{tabular}

Fonte: Dados da Pesquisa, 2019.

Nota: Médias seguidas por letras minúsculas nas linhas e maiúsculas nas colunas não diferem entre si pelo teste de Scott-Knott de probabilidade.

Os resultados apresentados na Tabela 9 demonstram que os tratamentos 7 e 14 dias foram os únicos que não apresentaram diferenciações entre si. Os demais tratamentos diferiram, porém na média final entre as sementes tratadas e não tratadas, os resultados não apresentaram estatisticamente diferença entre as sementes tratadas e não tratadas.

Estes resultados condizem com o estudo desenvolvido por Dan et al. (2011) em que os respectivos autores concluíram em seu experimento que o cultivo sob condições controladas, o acúmulo de MS durante o desenvolvimento inicial das plantas de soja não apresenta alterações em relação às sementes tratadas por inseticidas.
De acordo com um experimento realizado por Tavares et al (2013), a deficiência hídrica interferiu na fitomassa seca das plantas até o intervalo de 40 dias. Nesse sentido, o incremento da MS se mostrou inferior quando comparado com condições normais, nas quais a condição hídrica se apresentava dentro dos padrões considerados ideais para o desenvolvimento do cultivo. Além disso, o emprego de sementes de alta qualidade fisiológica foi responsável pela menor perda de MS e, por conseguinte, por maiores números de sementes e rendimento em condições de deficiência hídrica.

Conforme Machado (1988), o tempo de exposição no solo evidencia os danos 
que um patógeno pode provocar na semente. A partir dos achados deste estudo, é ratificada a reflexão do autor supracitado, uma vez que o tempo de exposição da semente ao solo impactou negativamente no poder germinativo das sementes e, por conseguinte, no stand final das plantas de soja. Este resultado em campo representa uma perda significativa em produtividade. Assim, o período compreendido entre 0 a 14 dias foi o que obteve melhores resultados, em função do fato de a semente estar menos exposta aos patógenos do solo.

Também, constata-se a influência de diversos fatores na qualidade das sementes, corroborando o trabalho desenvolvido por Carvalho (2012), em que o respectivo autor elenca alguns fatores que influenciam na qualidade das sementes, tais como, longevidade, condições climáticas como temperatura, água, oxigênio, grau de injúria mecânica sofrida

\section{CONCLUSÕES}

O déficit hídrico, até 14 dias, não impactou na germinação e rendimento produtivo das sementes. Porém, após esse período, observa-se que a escassez de da agua influenciará na qualidade fisiológica das sementes, comprometendo o stand final.

Em relação aos resultados advindos do tratamento de sementes, foi constatado que as sementes tratadas não influenciaram positivamente em ganhos produtivos e, por outro lado, as sementes não tratadas obtiveram melhores resultados. Esta situação evidenciada se traduz em uma aspecto que diverge do posicionamento de alguns autores, em que o tratamento das

\section{REFERÊNCIAS BIBLIOGRÁFICAS}

BARROS, R. G. et al. Compatibilidade do inseticida thiamethoxam com fungicidas utilizados no tratamento de sementes de feijoeiro. Santo Antônio do Goiás: Embrapa Arroz e Feijão, 2001. Disponível

em:<https://ainfo.cnptia.embrapa.br/digital pelas sementes e condições de armazenagem. No caso específico deste estudo, é possível observar injúrias decorrentes da inserção da mistura que comprometeram a qualidade fisiológica e química da semente tratada, influenciando no resultado final da pesquisa.

Assim, a partir dos dados obtidos, é possível relacionar os resultados menos favoráveis das sementes tratadas industrialmente com uma possível ineficácia do tratamento das respectivas sementes empregadas neste experimento.

Esta conclusão condiz com a reflexão de Barros et al. (2001), que afirmam que o tratamento bem-sucedido das sementes tratadas industrialmente depende da compatibilidade entre as informações sobre o produto, no que se refere a espectro de ação, toxicologia, efeitos fitotóxicos e compatibilidade com outros produtos.

sementes é considerado benéfico e de grande valia para a produtividade.

Essa divergência leva a crer que o tratamento empregado no experimento não foi realizado de forma adequada, causando fitotoxidez na semente ou injúrias causadas no momento da inserção da mistura que comprometeram a qualidade fisiológica e química da semente tratada.

Em função disso, este estudo demonstra que as empresas que preveem tratamento de sementes devem assegurar que o processo industrial empregado seja condizente com as características fisioquímicas dos defensivos empregados no tratamento.

/bitstream/item/28445/1/pat31n2Barros.pdf >. Acesso em: 09 maio 2019.

CAMPOS, R. J. Compatibilidade de uso de inoculante e fungicidas no tratamento de sementes de soja. Londrina: Embrapa Soja, 2000. Circular Técnica, ISSN 1516-7860, 
n. 26. Disponível em: $<$ https://www.embrapa.br/busca-depublicacoes//publicacao/446001/compatibilidade-deuso-de-inoculantes-e-fungicidas-notratamento-de-sementes-de-soja>. Acesso em: 10 jul., 2019.

CARVALHO, L. S. M. J. de. Desempenho de sementes de feijão tratadas com bioativador. 2012. 26f. Dissertação (Mestrado Profissional), Programa de PósGraduação em Ciência e Tecnologia de sementes. Universidade Federal de Pelotas, Pelotas, RS.

CUNHA, P. R. et al. Diferentes tratamentos de sementes sobre o desenvolvimento de plantas de soja Ciência Rural, v. 45, n. 10, out., 2015, p. 1761-1767. Universidade Federal de Santa Maria, Santa Maria. Disponível em: $<$ http://www.scielo.br/pdf/cr/v45n10/01038478-cr-cr20140742.pdf >. Acesso em: 10 jul., 2019.

DAN, L. G. M. et al. Tratamento de sementes com inseticida e a qualidade fisiológica de sementes de soja. Revista Caatinga, Mossoró, v. 25, n. 1, p. 45-51, jan./mar., 2012. Disponível em: $<$ https://periodicos.ufersa.edu.br/index.php /caatinga/article/download/2073/pdf>.

Acesso em: 10 jul., 2019.

Efeito de diferentes inseticidas sobre a qualidade fisiológica de sementes de soja. Gl. Sci. Technol., v. 3, n. 1, p. 5057, jan./abr., 2010. Disponível em: <https://www.researchgate.net/publication/ 287823502_Efeito_de_diferentes_inseticid as_sobre_a_qualidade_fisiologica_de_sem entes_de_soja>. Acesso em: 10 jul., 2019.

DIAS, D. C. F. S.; MARCOS FILHO, J. Testes de condutividade elétrica para avaliação do vigor de sementes de soja. (Glycine $\max$ (L.) Merrill). Revista Sci. Agric., v. 53, n. 1, Piracicaba, jun., abr., $1996 . \quad$ Disponível em: $<$ http://www.scielo.br/scielo.php?script=sc i_arttext\&pid=S0103-
90161996000100005>. Acesso em: 10 jun., 2019.

EMBRAPA SOJA. Veja o que esperar da safra de soja 2018/2019. Canal Rural, set., 2018. Disponível em: <https://blogs.canalrural.uol.com.br/embra pasoja/2018/09/04/o-que-esperar-da-safrade-soja-2018-19/>. Acesso em: 29 maio 2019.

FREITAS, M. C. M. de. A cultura da soja no Brasil: o crescimento da produção brasileira e o surgimento de uma nova fronteira agrícola. Enciclopédia Biosfera, v. 7, n. 12, Goiânia, 2011. Disponível em: <http://conhecer.org.br/enciclop/2011a/agr arias/a\%20cultura\%20da\%20soja.pdf $>$. Acesso em: 30 maio 2019.

GUEDES, R. S. et al. Testes de vigor na avaliação da qualidade fisiológica de sementes Erythrina velutina Willd. (FABACEAE - PAPILIONOIDEAE). Ciência Agrotec., v. 33, n. 5, Lavras, set./ out., 2009. Disponível em: <http://www.scielo.br/scielo.php?script=sc i_arttext\&pid=S1413-

70542009000500023>. Acesso em: 10 jul., 2019.

KROHN, N. G.; MALAVASI, M. M. Qualidade fisiológica de sementes de soja tratadas com fungicidas durante e após o armazenamento. Revista Brasileira de Sementes, v. 26, n. 2, p. 91-97, 2004. Disponível em: $<$ http://www.scielo.br/scielo.php?script=sc i_arttext\&pid=S0101-

31222004000200013>. Acesso em: 10 jul., 2019.

MACHADO, B. Q. V. et al. Germinação de cultivares de soja submetidas ao déficit hídrico induzido por polietinenoglicol. Cultura Agronômica, v. 25, n. 2, Ilha Solteira, p. 137-146, 2016. Disponível em: <http://ojs.unesp.br/index.php/rculturaagro nomica/article/view/2329/1762>. Acesso em: 30 maio 2019. 
MACHADO, J. C. Patologia de sementes: fundamentos e aplicações. Ministério da Educação, ESAL/FAEPE. Brasília: Ministério da Educação, Lavras, 1988. Disponível em: $<$ https://www.bing.com/search?q=machad $0 \% 2 \mathrm{C}+\mathrm{j} .+\mathrm{c} .+$ patologia+de+sementes $\% 3 \mathrm{~A}$ + fundamentos+e+aplica\% $\% 3 \% \mathrm{~A} 7 \% \mathrm{C} 3 \% \mathrm{~B}$ 5 es\&form $=$ EDGEAR\&qs $=P F \& c v i d=494 \mathrm{f}$ $19563 \mathrm{c} 8144969 \mathrm{fc} 8 \mathrm{~d} 5 \mathrm{c} 390 \mathrm{cb} 0 \mathrm{e} 0 \mathrm{f} \& \mathrm{cc}=\mathrm{BR}$ \&setlang=pt-BR\&PC=DCTS\#>. Acesso em: 03 maio 2019.

MARCOS FILHO, J. Importância do potencial fisiológico da semente de soja. Informativo Abrates, v. 23, n., abr., Londrina, 2013. Disponível em: <https://www.abrates.org.br/img/informati ons/950ff7fa-c03a-4960-a520-

f6cb0870babe_IA\%20vol.23\%20n.1.pdf\#p age $=21>$. Acesso em: 29 maio 2019 .

MILANESI, J. H. Adubação da cultura da soja baseada nos teores mínimos de fósforo e potássio no solo. 2015. 73 p. Dissertação (Mestrado em Agricultura de Precisão) - Universidade Federal de Santa Maria, Santa Maria, SP, 2015. Disponível em:

<http://w3.ufsm.br/ppgap/images/dissertac oes/2015/Jnior-Henrique-Milanesi.pdf $>$.

Acesso em: 30 maio 2019.

SILVA, A. C. da. et al. BATISTA, H. R. A importância da soja para o agronegócio brasileiro: uma análise sob o enfoque da produção, do emprego e exportação. In: V ENCONTRO DE ECONOMIA, 2011. Disponível em: <http://www.apec.unesc.net/V_EEC/sesso es_tematicas/Economia\%20rural\%20e\%20 agricultura\%20familiar/A\%20IMPORTÂN CIA\%20DA\%20SOJA\%20PARA $\% 200 \%$ 20AGRONEGÓCIO\%20BRASILEIRO.pd f>. Acesso em: 29 maio 2019.

TAVARES, L. C. et al. Desempenho de sementes de soja sob deficiência hídrica: rendimento e qualidade fisiológica da geração F1. Ciência Rural, v. 43, n. 8, ago., 2013, p. 1357-1363. Disponível em: $<$ http://www.scielo.br/scielo.php?script=sc
i_arttext\&pid=S0103-

84782013000800003>. Acesso em: 10 jul., 2019.

VIEIRA, F. C. F. et al. Aspectos fisiológicos e bioquímicos de cultivares de soja submetidos a déficit hídrico induzido por PEG 6000. Biosci. J., Uberlândia, v. 29, n. 2, p. 543-552, mar./abr. 2013. Disponível em: <https://www.researchgate.net/publication/ 270450410_Phisiological_and_biochemica 1_aspects_of_soybean_cultivars_submitted _to_water_deficit_induced_by_peg_6000> . Acesso em: 10 jul., 2019. 